\title{
Steady Flow of a Viscous Incompressible Fluid in an Unbounded «Funnel-Shaped» Domain $(*)$.
}

\author{
Arianna Passerini - M. Cristina Patria - Gudrun Thäter
}

Abstract. - In this paper we consider a domain $\Omega$ which is tube-like at one exit to infinity and the halfspace at the other side. We prove existence of steady motions for the Navier-Stokes problem and for the case in which the fluid is moving through a porous medium at rest filling $\Omega$. In both cases the proof holds for arbitrary fluxes. We describe the asymptotic behaviour of the solutions in the halfspace for both problems.

\section{1. - Introduction and problem formulation.}

Given $\boldsymbol{x} \in \mathbb{R}^{3}$, let $\boldsymbol{x}^{\prime} \equiv\left(x_{1}, x_{2}\right)$ be its projection onto the plane. We define the domain $\Omega$ and the section $\Sigma(l)$ as follows:

$$
\begin{aligned}
& \Omega \equiv\left\{\left(\boldsymbol{x}^{\prime}, x_{3}\right) \in \mathbb{R}^{3}:\left|\boldsymbol{x}^{\prime}\right| \leqslant R_{0} \text { or } x_{3} \leqslant 0\right\}, \\
& \Sigma(l) \equiv\left\{\left(\boldsymbol{x}^{\prime}, l\right) \in \mathbb{R}^{3}:\left|\boldsymbol{x}^{\prime}\right| \leqslant R_{0}\right\} .
\end{aligned}
$$

In the following, the problem under consideration is to find a velocity-field $v$ and a pressure $p$ such that

(PM)

$$
\begin{cases}-v \Delta v+\alpha^{2} \boldsymbol{v}+\boldsymbol{v} \cdot \nabla \boldsymbol{v}+\nabla p=0 & \\ \nabla \cdot \boldsymbol{v}=0, & \text { in } \Omega, \\ \left.\boldsymbol{v}\right|_{\partial \Omega}=0, & \\ \int_{\Sigma(0)} v_{\mathrm{s}}=\Phi . & \end{cases}
$$

(*) Entrata in Redazione il 28 novembre 1994.

Indirizzo degli AA.: A. PAsserini, M. CRIstina PATRia: University of Ferrara, Dipartimento di Matematica, Via Machiavelli 35, I-44100 Ferrara, Italy, e-mail: pat@dns.unife.it; G. THÄTER: University of Paderborn, Fachbereich Mathematik/Informatik, Warburger Straße 100, D-33098 Paderborn, Germany. 
Here by $v>0, \alpha, R_{0}$ and $\Phi$ are real constants denoting the kinetical viscosity, the extra viscous force, the radius of the tube and the flux, $\partial \Omega$ is the boundary of the domain.

We actually consider a regularization of $\partial \Omega$ in such a way that «angles» are avoided.

The equations (PM) describe the steady motion of a viscous incompressible fluid through a porous medium, occupying $\Omega$, that is at rest. The term $\alpha^{2} v$ is an approximation for the viscous interaction between the fluid and the medium. For $\alpha=0$ we get the usual steady Navier-Stokes equations and we denote this case with (NS).

In the background, there is the intention to use the results for considering the pollution transport with water through a sewer to the sea. The main question is, if the flux (as measurable parameter in the river) is given, what is predictable on the distribution of pollution in the sea, near the mouth, for different values of $\alpha$ and $\Phi$ ?

Therefore $\Omega$ consists of two main parts: the tube-like part for $x_{3}>0$ as model for the sewer and the halfspace for $x_{3}<0$ as representative of the ocean. The mouth lies at $x_{3}=0$.

The problem (PM) has already been studied in the halfspace by PASSERINI ([10]). In that paper existence and regularity of the solutions are proved and the asymptotic decay is estimated.

The linear problem associated to (PM) is nothing else than the resolvent problem for the Stokes system studied in [5].

The problem (NS) in $\mathbb{R}^{3}$ was studied in [3], [7], [4], [9], [2]. In particular in [4] the optimal decay is proved, for zero boundary datum and external body force of compact support.

Our paper is organized in the following way.

In Section 2 we recall the properties of a flux carrier field $\boldsymbol{a}$, prescribed as in [6], for the domain here considered. In Section 3 we show that the technique of LADYZHENSKAYA and SolonNIKov (from [8]), developed for infinite tubes, is useful for the tube-like part of $\Omega$. All together enables us to prove existence of weak solutions to $(\mathrm{PM})$ and (NS) in $\Omega$ for every flux $\Phi$ (Section 3 ).

For (NS) it is well known that there exist no solutions with finite Dirichlet integral $\left.{ }^{(}\right)$if $\Omega$ is the infinite cylinder. Defining for any $l \geqslant 0$

$$
\Omega_{l} \equiv\left\{\boldsymbol{x} \in \Omega:\left|x_{3}\right| \leqslant l\right\},
$$

the best possible behaviour of solutions to (NS) in the infinite cylinder is

$$
\int_{\Omega_{l}}|\nabla v|^{2}<c_{1} l+c_{2},
$$

(1) Dirichlet integral and Dirichlet norm are $\left(\int_{\Omega}|\nabla v|^{2}\right)^{1 / 2}=\|\nabla v\|_{2, \Omega}$. 
where $c_{1}, c_{2}$ are positive constants, $c_{1}=c_{1}\left(R_{0},|\Phi|, v\right), c_{2}=c_{2}(|\Phi|, v)\left({ }^{2}\right)$. This means, that the square of the Dirichlet norm grows linear with the $x_{3}$-direction. For details see [8].

In Section 3 it is shown, that the situation for (NS) and (PM) is the same if we consider domains of the form:

$$
\Omega_{l} \equiv\left\{\boldsymbol{x} \in \Omega: x_{3} \leqslant l\right\}
$$

Section 4 is devoted to the optimal decay of the solutions to both problems. Smallness assumptions on the flux are necessary for (NS) only. For (PM) the decay is as in [10]. For (NS) we follow the ideas of [4].

\section{2. - Definitions and preliminary results.}

The functions which appear can be scalar, vector or tensor valued functions according to the context. The following notation for function spaces will be used:

$$
\begin{aligned}
& \mathscr{O}(\Omega) \equiv\left\{\varphi \in C_{0}^{\infty}(\Omega): \nabla \cdot \varphi=0\right\}, \\
& H^{1}(\Omega) \equiv \overline{\mathscr{O}(\Omega)^{\|}} \cdot \|_{1,2, \Omega}, \\
& \mathscr{O}_{0}^{1,2}(\Omega) \equiv \overline{\mathscr{O}(\Omega)^{\|\nabla \cdot\|_{2, \Omega}} .}
\end{aligned}
$$

The space $\mathscr{O}_{0}^{1,2}(\Omega)$ is useful for (NS) and $H^{1}(\Omega)$ for (PM). in $\Omega$.

Here and in what follows $\|\cdot\|_{q, \Omega}$ is the $L^{q}$-norm and $(\cdot, \cdot)_{\Omega}$ the $L^{2}$-scalar product

All functions $\varphi$ that together with their first derivatives belong to $L^{2}(\Omega)$, form the space $W^{1,2}(\Omega)$ and $\|\varphi\|_{1,2, \Omega}^{2} \equiv \int_{\Omega}\left(\varphi^{2}+|\nabla \varphi|^{2}\right)$. The subspace of functions with zero traces on $\partial \Omega$ is denoted by $W_{0}^{1,2}(\Omega)$.

When unnecessary we omit the subscript for the domain in the symbols of the norm and the scalar product.

We shall prove the existence of solutions to problems (PM) and (NS) of the kind

( $\left.{ }^{2}\right)$ Here and in the sequel by $c\left(c_{1}, c_{2}\right)$ we denote a generic constant whose possible dependence on parameters will be specified when necessary. If its numerical value is unessential for our aims, then it may have several different values. 
$\boldsymbol{v}=\boldsymbol{u}+\boldsymbol{a}$, where $\boldsymbol{u}$ is searched as solution of

$(\mathrm{PM}) \begin{cases}-v \Delta \boldsymbol{u}+\alpha^{2} \boldsymbol{u}+\boldsymbol{u} \cdot \nabla \boldsymbol{u}+\boldsymbol{u} \cdot \nabla \boldsymbol{a}+\boldsymbol{a} \cdot \nabla \boldsymbol{u}+\nabla p=v \Delta \boldsymbol{a}-\alpha^{2} \boldsymbol{a}-\boldsymbol{a} \cdot \nabla \boldsymbol{a}, \\ \nabla \cdot \boldsymbol{u}=0, & \text { in } \Omega, \\ \left.\boldsymbol{u}\right|_{\partial \Omega}=0, & \\ \int_{\Sigma(0)} u_{3}=0, & \end{cases}$

and $\boldsymbol{a} \in C^{\infty}(\Omega)$ is a prescribed flux carrier function.

As in [6], $\boldsymbol{a}$ can be defined in the following way. Given $\boldsymbol{h}(x) \equiv\left(1 /\left(2 \pi\left|x^{\prime}\right|^{2}\right)\right)$. $\cdot\left(-x_{2}, x_{1}, 0\right)$ we set

$$
\boldsymbol{a} \equiv \Phi \nabla \times(\zeta \boldsymbol{h})=\Phi(\nabla \zeta \times \boldsymbol{h})
$$

where

$$
\zeta(\boldsymbol{x}) \equiv \Psi\left(\varepsilon \ln \frac{\sigma\left(\left|\boldsymbol{x}^{\prime}\right|\right)}{\varrho(\boldsymbol{x})}\right) .
$$

The functions $\Psi$ and $\sigma$, which are smooth and non decreasing, are such that

$$
\Psi(t)=\left\{\begin{array}{ll}
0 & \text { if } t \leqslant 0, \\
1 & \text { if } t \geqslant 1,
\end{array} \quad \sigma(t)= \begin{cases}R_{0} / 2 & \text { for } t<R_{0} / 2, \\
t & \text { for } t>R_{0},\end{cases}\right.
$$

$\varrho(\boldsymbol{x})$ is the regularized distance of $\boldsymbol{x}$ from $\partial \Omega$ and $\varepsilon \in(0,1)$ is an arbitrary constant. As a consequence of its definition, the vector field $\boldsymbol{a}$ is such that

(3)

$$
\left\{\begin{array}{l}
\operatorname{supp} \boldsymbol{a} \subset \operatorname{supp} \nabla \xi=\left\{\boldsymbol{x} \in \Omega: \varrho(\boldsymbol{x})<\sigma\left(\left|\boldsymbol{x}^{\prime}\right|\right)<e^{1 / \varepsilon} \varrho(\boldsymbol{x})\right\} \\
|\boldsymbol{a}(\boldsymbol{x})| \leqslant \frac{c|\Phi| \varepsilon}{\varrho(\boldsymbol{x})\left|\boldsymbol{x}^{\prime}\right|} \\
|\nabla \boldsymbol{a}(\boldsymbol{x})| \leqslant c|\Phi| \varepsilon^{2}\left(\frac{1}{\varrho(\boldsymbol{x})\left|\boldsymbol{x}^{\prime}\right|^{2}}+\frac{1}{\varrho^{2}(\boldsymbol{x})\left|\boldsymbol{x}^{\prime}\right|}\right)
\end{array}\right.
$$

Thus, as $|\boldsymbol{x}| \rightarrow \infty$, for $x_{3}<0$

$$
|\boldsymbol{a}(\boldsymbol{x})|=O\left(|\boldsymbol{x}|^{-2}\right), \quad|\nabla \boldsymbol{a}(\boldsymbol{x})|=O\left(|\boldsymbol{x}|^{-3}\right), \quad|\Delta \boldsymbol{a}(\boldsymbol{x})|=O\left(|\boldsymbol{x}|^{-4}\right) .
$$

Moreover, for $x_{3}>0 a$ is bounded and does not depend on $x_{3}$.

Finally, the following properties hold:

(i) $\nabla \cdot \boldsymbol{a}=0,\left.\boldsymbol{a}\right|_{\partial \Omega}=0, \int_{\Sigma(0)} a_{3}=\Phi$,

(ii) $\int_{\Omega} w^{2} a^{2}<\varepsilon^{2} \Phi^{2} \cdot c \int_{\Omega}|\nabla w|^{2}, \forall w \in \mathcal{O}(\Omega), c=c\left(R_{0}\right)$. 
The proof of these properties is completely analogous to that given in [6] for the halfspace and the aperture domain. In particulr, in order to obtain (ii) in the pipe it is sufficient to apply the Poincaré inequality.

We note that (ii) is true also when the domain of integration is $\Omega_{l}, \forall l \geqslant 0$.

At this stage we are able to prove an existence result for the weak formulation of the problem (1) in $\Omega_{l}$.

LEMMA 1. - For fixed $l \in[0,+\infty)$ and $\boldsymbol{a}$ as defined in (2) there exists a solution $\boldsymbol{u}^{l}$ to the problem

$$
\left\{\begin{array}{c}
v(\nabla \boldsymbol{u}, \nabla \varphi)_{\Omega_{l}}+(\boldsymbol{u} \cdot \nabla \boldsymbol{u}, \varphi)_{\Omega_{l}}+(\boldsymbol{a} \cdot \nabla \boldsymbol{u}, \varphi)_{\Omega_{l}}+(\boldsymbol{u} \cdot \nabla \boldsymbol{a}, \varphi)_{\Omega_{l}}+\alpha^{2}(\boldsymbol{u}, \varphi)_{\Omega_{l}}= \\
\quad=(-v \nabla \boldsymbol{a}+\boldsymbol{a} \otimes \boldsymbol{a}, \nabla \varphi)_{\Omega_{l}}-\alpha^{2}(\boldsymbol{a}, \varphi)_{\Omega_{l}}, \quad \forall \varphi \in \mathcal{O}\left(\Omega_{l}\right), \\
(\boldsymbol{u}, \nabla \Psi)_{\Omega_{l}}=0, \quad \forall \Psi \in C_{0}^{\infty}\left(\Omega_{l}\right),
\end{array}\right.
$$

such that $\boldsymbol{u}^{l} \in \dot{\sigma}_{0}^{1,2}\left(\Omega_{l}\right)$ if $\alpha=0, \boldsymbol{u}^{l} \in H^{1}\left(\Omega_{l}\right)$ if $\alpha \neq 0$. Moreover

$$
\left\|\nabla \boldsymbol{u}^{l}\right\|_{2, \Omega_{l}}^{2} \leqslant c_{1} l+c_{2} \quad \text { if } \alpha=0, \quad\left\|\boldsymbol{u}^{l}\right\|_{1,2, \Omega_{l}}^{2} \leqslant c_{1} l+c_{2}, \quad \text { if } \alpha \neq 0,
$$

with $c_{1}=c_{1}\left(R_{0},|\Phi|, v\right), c_{2}=c_{2}(|\Phi|, v)$, independent of $l$.

Proof. - In order to show the existence of $\boldsymbol{u}^{l}$ we apply the wellknown Galerkin method (see [6]). It is easy to prove the existence of a sequence of approximating solutions $\left\{\boldsymbol{u}_{n}^{l}\right\}_{n \in \mathbb{N}}$ belonging to $n$-dimensional subspaces of $\mathscr{O}\left(\Omega_{l}\right)$. Moreover, from (i) and (ii) rewritten for $\Omega_{l}$ we can easily deduce the following estimates for $\boldsymbol{u}_{n}^{l}$

$$
\begin{cases}\left\|\nabla \boldsymbol{u}_{n}^{l}\right\|_{2, \Omega_{l}}^{2} \leqslant c \int_{\Omega_{l}}\left(|\nabla \boldsymbol{a}|^{2}+\boldsymbol{a}^{4}\right) & \text { for } \alpha=0 \\ \left\|\boldsymbol{u}_{n}^{l}\right\|_{1,2, \Omega_{l}}^{2} \leqslant c \int_{\Omega_{l}}\left(|\nabla \boldsymbol{a}|^{2}+\boldsymbol{a}^{2}+\boldsymbol{a}^{4}\right) & \text { for } \alpha \neq 0\end{cases}
$$

Since the constant $c$ does not depend on $n$, it is sufficient that the right-hand sides of (7) are finite to have the existence of a solution $\boldsymbol{u}^{l}$ to (5).

Properties (4) imply that the integrals on the right-hand side of (7) are finite for that part of $\Omega_{l}$ lying in the halfspace. On the other hand the estimates (3) do not depend on $x_{3}$ if $x_{3}>0$. Thus, we get

$$
\int_{0}^{l} \int_{\Sigma\left(x_{3}\right)}\left(|\nabla \boldsymbol{a}|^{2}+\boldsymbol{a}^{4}+\boldsymbol{a}^{2}\right) \leqslant d_{1} l,
$$

where $d_{1}$ is a constant depending on $R_{0}$ and $|\Phi|$. Therefore the final estimate is

$$
\int_{\Omega_{l}}\left(|\nabla \boldsymbol{a}|^{2}+\boldsymbol{a}^{4}+\boldsymbol{a}^{2}\right) \leqslant d_{1} l+d_{2}<\infty
$$


where $d_{2}$ is a constant depending on $R_{0}$ and $|\Phi|$. Notice that, after estimating with the aid of (ii), one has $c_{1}>d_{1} / \nu$ and $c_{2}>d_{2} / \nu$.

REMARK 1. - By well known results on decomposition of homogeneous Sobolev spaces we also get the existence of a pressure field $p^{l} \in L^{2}\left(\Omega_{l}\right), \forall l \geqslant 0$ (see [6]).

REMARK 2. - For any $\alpha, \boldsymbol{u}^{l} \in L^{6}\left(\Omega_{l}\right)(l \geqslant 0)$. This holds true because $\boldsymbol{u}^{l} \in L^{6}\left(\mathbb{R}_{-}^{3}\right)$ (see [4], Lemma 5) and $\boldsymbol{u}^{l} \in L^{6}\left(\Omega_{0, l}\right)$, where $\Omega_{k, l} \equiv \Omega_{l} \backslash \overline{\Omega_{k}}(k \leqslant l)$.

REMARK 3. - Since $\partial \Omega$ is regular, $\boldsymbol{u}^{l}$ and $p^{l}$ are $C^{\infty}(K)$ for any $K$ such that $\bar{K} \subset \Omega, \bar{K}$ compact.

REMARK 4. - If we look at (8) and recall that $\boldsymbol{v}=\boldsymbol{u}+\boldsymbol{a}$ it is clear that we cannot get, with the same procedure, a solution in the whole domain $\Omega$ having finite Dirichlet integral but at most

$$
\int_{\Omega_{l}}|\nabla v|^{2} \leqslant c_{1} l+c_{2}, \quad \forall l \geqslant 0 .
$$

Now, in order to prove the existence of a weak solution $v$ in $\Omega$ we want to show that

$$
\begin{cases}\left\|\nabla \boldsymbol{u}^{l}\right\|_{2, \Omega_{k}}^{2} \leqslant c_{1} k+c_{2}, & \forall k \in[0, l], \alpha=0, \\ \left\|\boldsymbol{u}^{l}\right\|_{1,2, \Omega_{k}}^{2} \leqslant c_{1} k+c_{2}, & \forall k \in[0, l], \alpha \neq 0 .\end{cases}
$$

First, in the case $\alpha=0$, we prove for the solution $\boldsymbol{u}^{l}$ found in Lemma 1 , the following «energy» inequality.

Lemma 2. - Let $\boldsymbol{u}^{l} \in \mathscr{O}_{0}^{1,2}\left(\Omega_{l}\right)$ be a solution to (5) for $\alpha=0$. Then $\forall k \in[0, l]$

$$
\begin{aligned}
& \nu\left\|\nabla \boldsymbol{u}^{l}\right\|_{2, \Omega_{k}}^{2} \leqslant\left(\boldsymbol{u}^{l} \cdot \nabla \boldsymbol{u}^{l}, \boldsymbol{a}\right)_{\Omega_{k}}+\left(\nabla \boldsymbol{u}^{l}, \boldsymbol{a} \otimes \boldsymbol{a}-\nu \nabla \boldsymbol{a}\right)_{\Omega_{k}}+ \\
& \quad+\int_{\Sigma(k)}\left(\frac{\nu \partial}{\partial x_{3}}\left(\boldsymbol{u}^{l}+\boldsymbol{a}\right) \cdot \boldsymbol{u}^{l}-p^{l} u_{3}^{l}-\frac{1}{2}\left(\boldsymbol{u}^{l}\right)^{2}\left(a_{3}+u_{3}^{l}\right)-a_{3}\left(\boldsymbol{a} \cdot \boldsymbol{u}^{l}\right)-u_{3}^{l}\left(\boldsymbol{a} \cdot \boldsymbol{u}^{l}\right)\right) .
\end{aligned}
$$

Proof. - Let us indicate by $\boldsymbol{u}_{n}^{l}$ the Galerkin approximations for $\boldsymbol{u}^{l}$. They verify

$$
v\left(\nabla \boldsymbol{u}_{n}^{l}, \nabla \boldsymbol{u}_{n}^{l}\right)_{\Omega_{l}}=\left(\boldsymbol{u}_{n}^{l} \cdot \nabla \boldsymbol{u}_{n}^{l}, \boldsymbol{a}\right)_{\Omega_{l}}+\left(\nabla \boldsymbol{u}_{n}^{l}, \boldsymbol{a} \otimes \boldsymbol{a}-\nu \nabla \boldsymbol{a}\right)_{\Omega_{l}} .
$$

On the other hand, from standard techniques for the convergence of the nonlinear term (see [6]), we have, for a subsequence $\boldsymbol{u}_{n^{\prime}}^{l}$,

$$
\lim _{n^{\prime} \rightarrow+\infty}\left(\boldsymbol{u}_{n^{\prime}}^{l} \cdot \nabla \boldsymbol{u}_{n^{\prime}}^{l}, \boldsymbol{a}\right)_{\Omega_{l}}=\left(\boldsymbol{u}^{l} \cdot \nabla \boldsymbol{u}^{l}, \boldsymbol{a}\right)_{\Omega_{l}} .
$$


Since $\nabla \boldsymbol{u}_{n^{\prime}}^{l} \rightarrow \nabla \boldsymbol{u}^{l}$ weakly,

$$
v\left\|\nabla \boldsymbol{u}^{l}\right\|_{2, \Omega_{l}}^{2} \leqslant \lim _{n^{\prime} \rightarrow+\infty} v\left\|\nabla \boldsymbol{u}_{n^{\prime}}^{l}\right\|_{2, \Omega_{l}}^{2}
$$

so that, by choosing $n=n^{\prime}$ and taking the limit of the right-hand side of (11), we arrive at

$$
v\left\|\nabla \boldsymbol{u}^{l}\right\|_{2, \Omega_{l}}^{2} \leqslant\left(\boldsymbol{u}^{l} \cdot \nabla \boldsymbol{u}^{l}, \boldsymbol{a}\right)_{\Omega_{l}}+\left(\boldsymbol{a} \otimes \boldsymbol{a}-v \nabla \boldsymbol{a}, \nabla \boldsymbol{u}^{l}\right)_{\Omega_{l}} .
$$

Consider now the bounded domain $\Omega_{k, l}=\Omega_{l} \backslash \overline{\Omega_{k}}$. We multiply (1) by $\boldsymbol{u}^{l}$ and integrate by parts over $\Omega_{k, l}$, we get

$$
\begin{aligned}
& v\left\|\nabla \boldsymbol{u}^{l}\right\|_{2, \Omega_{k, l}}^{2}=\left(\boldsymbol{u}^{l} \cdot \nabla \boldsymbol{u}^{l}, \boldsymbol{a}\right)_{\Omega_{k, l}}+\left(\boldsymbol{a} \otimes \boldsymbol{a}-\nu \nabla \boldsymbol{a}, \nabla \boldsymbol{u}^{l}\right)_{\Omega_{k, l}}+ \\
& -\int_{\Sigma(k)} \underbrace{\left(\frac{\nu \partial}{\partial x_{3}}\left(\boldsymbol{u}^{l}+\boldsymbol{a}\right) \cdot \boldsymbol{u}^{l}-p^{l} u_{3}^{l}-\frac{1}{2}\left(\boldsymbol{u}^{l}\right)^{2}\left(a_{3}+u_{3}^{l}\right)-a_{3}\left(\boldsymbol{a} \cdot \boldsymbol{u}^{l}\right)-u_{3}^{l}\left(\boldsymbol{a} \cdot \boldsymbol{u}^{l}\right)\right)}_{(\mathrm{S} l)} .
\end{aligned}
$$

Equation (13) can be obtained in this way because of Remark 3.

If we substract (13) from (12) we obtain the desired result (10).

REMARK 5. - We note that, for $k=l$, the surface integral in (10) vanishes.

REMARK 6. - If $\alpha \neq 0$ (problem (PM)), (10) must be substituted by

$$
\begin{aligned}
v\left\|\nabla \boldsymbol{u}^{l}\right\|_{2, \Omega_{k}}^{2}+\alpha^{2}\left\|\boldsymbol{u}^{l}\right\|_{2, \Omega_{k}}= & \\
& =\left(\boldsymbol{u}^{l} \cdot \nabla \boldsymbol{u}^{l}, \boldsymbol{a}\right)_{\Omega_{k}}+\left(\boldsymbol{a} \otimes \boldsymbol{a}-\nu \nabla \boldsymbol{a}, \nabla \boldsymbol{u}^{l}\right)_{\Omega_{k}}-\alpha^{2}\left(\boldsymbol{a}, \boldsymbol{u}^{l}\right)_{\Omega_{k}}+\int_{\Sigma(k)}(S I) .
\end{aligned}
$$

and hence, setting $v^{\prime}=\min \left\{v, \alpha^{2}\right\}$

$$
v^{\prime}\left\|\nabla \boldsymbol{u}^{l}\right\|_{1,2, \Omega_{k}}^{2} \leqslant\left(\boldsymbol{u}^{l} \cdot \nabla \boldsymbol{u}^{l}, \boldsymbol{a}\right)_{\Omega_{k}}+\left(\boldsymbol{a} \otimes \boldsymbol{a}-\nu \nabla \boldsymbol{a}, \nabla \boldsymbol{u}^{l}\right)_{\Omega_{\Omega_{k}}}-\alpha^{2}\left(\boldsymbol{a}, \boldsymbol{u}^{l}\right)_{\Omega_{k}}+\int_{\Sigma(k)}(S I) .
$$

In fact, taking into account Remark 3 and what is proved in [10], by considering $\Omega_{l}=\Omega_{0} \cup \Omega_{0, l}$, it can be deduced

$$
\begin{array}{lll}
\boldsymbol{u}^{l} \in W^{2, q}\left(\Omega_{l}\right), & \forall q \in(3 / 2,+\infty) & \text { if } \Phi \neq 0, \\
\boldsymbol{u}^{l} \in W^{2, q}\left(\Omega_{l}\right), & \forall q \in(1,+\infty) & \text { if } \Phi=0 .
\end{array}
$$

Thus, if we multiply the first equation of (1) by $\boldsymbol{u}^{l}$ and integrate over

$$
\Omega_{k}^{r} \equiv \Omega_{k} \cap\left\{\boldsymbol{x} \in \mathbb{R}_{-}^{3}:|\boldsymbol{x}| \leqslant r\right\},
$$

a diverging sequence of radii $\left\{r_{n}\right\}_{n \in \mathbb{N}}$ exists on which the surface integral tends to zero.

So, (14) is easily proved. 
At this stage, let us recall the following result, consequence of Lemma 2.3 in [8]:

LemMa 3. - Assume that the nonnegative smooth function $z(t)$, not identically equal to zero, satisfies on the segment $[1, T]$ the inequalities

$$
\begin{aligned}
& z(t) \leqslant A\left(z^{\prime}(t)+z^{\prime}(t)^{3 / 2}\right)+(B t+C)(1-\delta), \quad \delta \in(0,1), \\
& C \geqslant \frac{1}{\delta} A\left(B+B^{3 / 2}\right),
\end{aligned}
$$

where $A, B, C, \delta$ are positive constants. In this case, if

$$
z(T) \leqslant B \cdot T+C, \quad \text { then } z(t) \leqslant B \cdot t+C, \quad \forall t \in[1, T] .
$$

Finally we are able to prove the inequalities (9) and hence the existence theorem. To this we devote the next section.

\section{3. - Existence of a weak solution.}

Let us begin by proving the inequalities (9).

LEMMA 4. - Let $\boldsymbol{u}^{l}$ be the solution to (5) in $\Omega_{l}, \boldsymbol{u}^{l} \in \mathscr{O}_{0}^{1,2}\left(\Omega_{l}\right)$ if $\alpha=0, \boldsymbol{u}^{l} \in H^{1}\left(\Omega_{l}\right)$ if $a \neq 0$. Then, for all $k \in[0, l]$ we have

$$
\begin{cases}\left\|\nabla \boldsymbol{u}^{l}\right\|_{2, \Omega_{k}}^{2} \leqslant c_{1} k+c_{2}^{\prime} & \text { if } \alpha=0, \\ \left\|\boldsymbol{u}^{l}\right\|_{1,2, \Omega_{k}}^{2} \leqslant c_{1} k+c_{2}^{\prime} & \text { if } \alpha \neq 0,\end{cases}
$$

where $c_{1}=c_{1}\left\{R_{0},|\Phi|, \nu\right)$ and $c_{2}^{\prime}=c_{2}^{\prime}\left(R_{0},|\Phi|, \nu\right)$ are constants independent of $k$ and $l$.

PRoof. - For the sake of brevity we indicate both squared norms in (15) with $\chi(k)$. $\chi$ is clearly an increasing function of $k$ and is nonnegative.

For all $k \in[1, l]$ we define

$$
z(k) \equiv \int_{k-1}^{k} \chi(j) d j
$$

such that

$$
\chi(k-1) \leqslant z(k) \leqslant \chi(k), \quad \forall k \in[1, l] .
$$

Now we consider the energy inequalities (10) if $\alpha=0,(14)$ if $\alpha \neq 0$ and estimate the integrals at the right-hand side. We treat only the case $\alpha \neq 0$, the other case is completely analogous. 
By means of the Cauchy inequality and (ii) we easily obtain

$$
\chi(j) \leqslant c\left(\|\nabla \boldsymbol{a}\|_{2, \Omega_{j}}^{2}+\|\boldsymbol{a}\|_{4, \Omega_{j}}^{4}+\|\boldsymbol{a}\|_{2, \Omega_{j}}^{2}\right)+c_{0} \int_{\Sigma(j)}(S I) d \Sigma .
$$

Integrating both members of (17) from $k-1$ to $k, 1 \leqslant k \leqslant l$, and bearing in mind the properties of $\boldsymbol{a}$, we arrive at

$$
z(k) \leqslant c_{1} k+c_{2}+c_{0} \int_{\Omega_{k-1}, k}(S I) d \Omega .
$$

Now we have to estimate the integral in (18). To this end, we proceed as in [8] and use inequality (2.27) of [8]:

$$
\int_{\Omega_{k-1, k}}\left|\boldsymbol{u}^{l}\right|^{4} \leqslant c\left(\int_{\Omega_{k-1, k}}\left|\nabla \boldsymbol{u}^{l}\right|^{2}\right)^{2},
$$

which holds for any $\boldsymbol{u}^{l}$ vanishing on the lateral surface of $\Omega_{k-1, k}$. Moreover, using this inequality, Cauchy-Schwarz and the Poincaré inequalities together with (ii) and (3), after some calculations we arrive at

$$
\left\{\begin{array}{l}
\left|\int_{\Omega_{k-1, k}}\left(v \frac{\partial \boldsymbol{u}^{l}}{\partial x_{3}} \boldsymbol{u}^{l}+\left(\boldsymbol{a} \cdot \boldsymbol{u}^{l}\right) u_{3}^{l}+\frac{1}{2}\left(\boldsymbol{u}^{l}\right)^{2} a_{3}\right)\right| \leqslant c \int_{\Omega_{k-1, k}}\left|\nabla \boldsymbol{u}^{l}\right|^{2}, \\
\left|\int_{\Omega_{k-1, k}}\left(v \frac{\partial \boldsymbol{u}}{\partial x_{3}} \boldsymbol{u}^{l}-\left(\boldsymbol{a} \cdot \boldsymbol{u}^{l}\right) a_{3}\right)\right| \leqslant c \int_{\Omega_{k-1, k}}\left|\nabla \boldsymbol{u}^{l}\right|^{2}+c_{0}, \\
\left|\int_{\Omega_{k-1, k}} \frac{1}{2}\left(\boldsymbol{u}^{l}\right)^{2} u_{3}^{l}\right| \leqslant c\left(\int_{\Omega_{k-1, k}} \frac{1}{2}\left|\nabla \boldsymbol{u}^{l}\right|^{2}\right)^{3 / 2}
\end{array}\right.
$$

The pressure term $p^{l} u_{3}^{l}$ needs some attention.

Consider the problem

$$
\left\{\begin{array}{l}
\nabla \cdot f=u_{3}^{l} \quad \text { in } \Omega_{k-1, k}, \\
\left.f\right|_{\partial \Omega_{k-1, k}}=0 .
\end{array}\right.
$$

Since the compatibility condition (see [1]) $\int_{\Omega_{k-1, k}} u_{3}^{k}=0$ is automatically satisfied because of $\int_{\Sigma(j)} u_{3}^{l}=0, \forall j \in[k-1, k]$, problem $\stackrel{\Omega_{k-1, k}}{(20)}$ admits a solution $f \in W_{0}^{1,2}\left(\Omega_{k-1, k}\right)$ such
that

$$
\|\nabla f\|_{2, \Omega_{k-1, k}}^{2} \leqslant c\left\|u_{3}^{l}\right\|_{2, \Omega_{k-1, k}}^{2} .
$$


Using the same procedure as in [8] we get

$$
\int_{\Omega_{k-1, k}} p^{l} u_{3}^{l} \leqslant c\left(\int_{\Omega_{k-1, k}}\left|\nabla \boldsymbol{u}^{l}\right|^{2}+\left(\int_{\Omega_{k-1, k}}\left|\nabla \boldsymbol{u}^{l}\right|^{2}\right)^{3 / 2}\right)+c_{0} .
$$

Thus, (18) becomes

$$
z(k) \leqslant c_{1} k+c_{2}+c\left(\int_{\Omega_{k-1, k}}\left|\nabla \boldsymbol{u}^{l}\right|^{2}+\left(\int_{\Omega_{k-1, k}}\left|\nabla \boldsymbol{u}^{l}\right|^{2}\right)^{3 / 2}\right) .
$$

Therefore, due to the definition of $z$, we finally arrive at

$$
z(k) \leqslant c_{1} k+c_{2}+c\left(z^{\prime}(k)+z^{\prime}(k)^{3 / 2}\right), \quad \forall k \in[1, l] .
$$

On the other hand, using (16) and the estimate of Lemma 1, we have

$$
z(l) \leqslant \chi(l) \leqslant c_{1} l+c_{2} .
$$

In this inequality and in (21), $c_{2}$ can be inereased with an arbitrary positive constant $c_{2}^{\prime}>c_{2}$, such that $c_{2}^{\prime}(1-\delta)^{-1} \geqslant c \delta^{-1}\left(c_{1}(1-\delta)^{-1}+c_{1}^{3 / 2}(1-\delta)^{-3 / 2}\right)$.

Thus all the hypotheses of Lemma 3 are satisfied choosing $A=c, B=\epsilon_{1} /(1-\delta)$, $C=c_{2}^{\prime} /(1-\delta)$, so that

$$
z(k) \leqslant c_{1} k+c_{2}^{\prime}, \quad \forall k \in[1, l] .
$$

Now, the thesis follows from (16) and (22).

REMARK 7. - We note that $c_{1}, c_{2}, c$ in (21) and $c_{2}^{\prime}$ are polynomials, without the constant term, of degree 4 and 10 , respectively.

REMARK 8. - Inequalities (15) still hold if $k>l$ by extending $\boldsymbol{u}^{l}$ with zero for $x_{3}>l$.

Now we have all the tools to prove the main result of this section.

THEOREM 1. - Probiems (PM) and (NS) for $\Omega$ admit a weak solution $v$ such that, for any $l \geqslant 0$

$$
\begin{cases}\|\nabla \boldsymbol{v}\|_{2, \Omega_{l}}^{2} \leqslant c_{1} l+c_{2}^{\prime} & \text { if } \alpha=0, \\ \|\boldsymbol{v}\|_{1,2, \Omega_{l}}^{2} \leqslant c_{1} l+c_{2}^{\prime} & \text { if } \alpha \neq 0,\end{cases}
$$

where $c_{1}=c_{1}\left(R_{0}, \nu,|\Phi|\right), c_{2}^{\prime}=c_{2}^{\prime}\left(R_{0}, v,|\Phi|\right)$. 
Proof. - We take $\boldsymbol{v}=\boldsymbol{u}+\boldsymbol{a}$, with $\boldsymbol{a}$ from (2). In order to construct $\boldsymbol{u}$ in whole $\Omega$ we consider a family of subdomains

$$
\Omega_{0} \subset \Omega_{1} \subset \Omega_{2} \subset \ldots \text { such that } \bigcup_{i=0}^{\infty} \Omega_{i}=\Omega .
$$

Let $k>0$ and consider for all $l$ the solution $\boldsymbol{u}^{l}$ to (5) in $\Omega_{l}$. Lemma 4 and Remark 8 ensure that

$$
\left\|\nabla \boldsymbol{u}^{l}\right\|_{2, \Omega_{k}}^{2} \leqslant c_{1} k+c_{2}^{\prime} \quad \text { if } \alpha=0, \quad\left\|\boldsymbol{u}^{l}\right\|_{1,2, \Omega_{k}}^{2} \leqslant c_{1} k+c_{2}^{\prime} \quad \text { if } \alpha \neq 0 .
$$

So $\left\{u^{l}\right\}_{l \in \mathbb{N}}$ is a sequence of vector fields whose gradient is uniformly bounded in $L^{2}\left(\Omega_{k}\right)$. Therefore there exists a subsequence $\left\{u_{k}^{l}\right\}_{j \in \mathbb{N}}$ converging weakly in the norm of the gradient.

Then consider $\Omega_{k+1}$. It holds

$$
\left\|\nabla \boldsymbol{u}_{k}^{l_{k}}\right\|_{2, \Omega_{k+1}}^{2} \leqslant c_{1}(k+1)+c_{2}^{\prime} \quad \text { if } \alpha=0, \quad\left\|\boldsymbol{u}_{k}^{l_{k}}\right\|_{1,2, \Omega_{k+1}}^{2} \leqslant c_{1}(k+1)+c_{2}^{\prime} \quad \text { if } \alpha \neq 0 .
$$

We can extract a new subsequence $\left\{\boldsymbol{u}_{k+1}^{l}\right\}_{j \in \mathbb{N}}$ from the previous one such that it converges in $\Omega_{k+1}$. Since $L^{2}\left(\Omega_{k}\right) \subset L^{2}\left(\Omega_{k+1}\right)$, for the uniqueness of the limit, the limits of $\left\{\boldsymbol{u}_{k}^{l_{j}}\right\}_{j \in \mathbb{N}}$ and $\left\{\boldsymbol{u}_{k+1}^{l_{j}}\right\}_{j \in \mathbb{N}}$ coincide in $\Omega_{k}$. And so on.

A classical procedure of diagonalization furnishes the final sequence whose limit $\boldsymbol{u}$ satisfies (1) and is such that (23) holds.

\section{4. - Asymptotic decay in the halfspace.}

In this section we establish the asymptotic behaviour of the solution $\boldsymbol{v}$ as $|\boldsymbol{x}| \rightarrow+$ $+\infty$ for $x_{3}<0$. To this end, we will deal with restrictions of these solutions to $\mathbb{R}_{-}^{3}$. Every restriction will be indicated with the same symbol as the solution in $\Omega$.

Case $\alpha=0$ : We must suitably improve ther esults of [4] concerning the NavierStokes problem in the halfspace, with compact support body force and zero boundary data, to take into account non-zero boundary data. To this end, we will transform our problem into a new one with zero boundary data and non-zero body force $F$. However, the difficulty here is that, unlike[4], $F$ is not of compact support.

$$
\begin{aligned}
& \text { Since } \int_{\Sigma(0)} u_{3}=0 \text { holds true, we can write } \\
& \qquad \boldsymbol{u}=\boldsymbol{u}_{0}+\boldsymbol{w},
\end{aligned}
$$

where the trace $\boldsymbol{u}_{0}^{*}$ of $\boldsymbol{u}_{0}$ on $\Sigma(0)$ is zero and $\boldsymbol{w}$ has compact support and zero flux through $\Sigma(0)$.

We will describe how to construct $\boldsymbol{w}$, the extension of the boundary data of $\boldsymbol{u}$ on $\Sigma(0)$ 
Let $\boldsymbol{u}^{*}$ be the trace of $\boldsymbol{u}$ on $\Sigma(0)$ and $\Omega^{\prime} \subset \mathbb{R}_{-}^{3}$ a bounded regular domain, such that $\Sigma(0) \subset \partial \Omega^{\prime} \cap \partial R_{-}^{3}$. Then, we can choose $\boldsymbol{w}$ as the solution of the auxiliary Stokes problem

$$
\begin{aligned}
& -v \Delta \boldsymbol{w}+\nabla \tau=0, \quad \nabla \cdot \boldsymbol{w}=0 \quad \text { in } \Omega^{\prime} \\
& \left.\boldsymbol{w}\right|_{\Sigma(0)}=\boldsymbol{u}^{*} \\
& \left.\boldsymbol{w}\right|_{\partial \Omega^{\prime} \backslash \Sigma(0)}=0 .
\end{aligned}
$$

The solution $(\boldsymbol{w}, \tau)$ verifies

$$
\|\nabla \boldsymbol{w}\|_{2, \Omega^{\prime}}+\|\tau\|_{2, \Omega^{\prime}} \leqslant c\left\|\boldsymbol{u}^{*}\right\|_{1 / 2,2, \partial \Omega^{\prime}},
$$

where the norm on the right-hand side is the usual one for trace spaces (see [6], Chapter 2, Section 3).

Moreover, since the data on the boundary are $C^{\infty}\left(\partial \Omega^{\prime}\right)$, the solution $(\boldsymbol{w}, \tau)$ belongs to $C^{\infty}\left(\Omega^{\prime}\right)$.

Clearly, $\boldsymbol{w}$ can be extended to be zero outside of $\Omega^{\prime}$ in $\mathbb{R}_{-}^{3}$. The function in $C_{0}^{\infty} \overline{\left(\mathbb{R}_{-}^{3}\right)}$ retains the notation $\boldsymbol{w}$.

Recalling that $\boldsymbol{v}=\boldsymbol{u}+\boldsymbol{a}$, we get

$$
v=u_{0}+b
$$

with

$$
b=a+w .
$$

If $x_{3}<0$, and for $|\boldsymbol{x}|$ sufficiently large, $\boldsymbol{b}$ behaves as $|\boldsymbol{x}|^{-2}$, because $\boldsymbol{w}$ has compact support and (4) holds. Thus, we have to check the asymptotic behaviour of $\boldsymbol{u}_{0}$ only.

Setting

$$
G=\nu \nabla b-b \otimes b-u_{0} \otimes b-b \otimes u_{0}, \quad F=\nabla \cdot G,
$$

we can state that $\boldsymbol{u}_{0} \in \mathscr{O}_{0}^{1,2}\left(\mathbb{R}_{-}^{3}\right)$ verifies

$$
\left\{\begin{array}{l}
-v \Delta \boldsymbol{u}_{0}+\boldsymbol{u}_{0} \cdot \nabla \boldsymbol{u}_{0}+\nabla \pi=F, \quad \nabla \cdot \boldsymbol{u}_{0}=0 \quad \text { in } \mathbb{R}_{-}^{3}, \\
\left.\boldsymbol{u}_{0}\right|_{\partial \mathbb{R}_{-}^{3}}=0
\end{array}\right.
$$

In order to obtain the desired decay, we need to deduce from (25) the properties of $F$.

We start by defining for $q \in(1, \infty)$

$$
D_{0}^{1, q}\left(\mathbb{R}_{-}^{3}\right) \equiv{\overline{C_{0}^{\infty}\left(\mathbb{R}_{-}^{3}\right)}}^{\|\nabla \cdot\|_{q, \mathbb{R}^{3}}} .
$$

Denoting the Hölder conjugate of $q$ with $q^{\prime}, D_{0}^{-1, q^{\prime}}\left(\mathbb{R}_{-}^{3}\right)$ is defined as the dual space of $D_{0}^{1, q}\left(\mathbb{R}_{-}^{3}\right)$. As known, the class of functionals on $D_{0}^{1, q}\left(\mathbb{R}_{-}^{3}\right)$ of the form

$$
\mathscr{F}(\varphi)=\int_{\mathbb{R}_{-}^{3}} f \varphi, \quad f \in C_{0}^{\infty}\left(\mathbb{R}_{-}^{3}\right)
$$


is dense in $D_{0}^{-1, q^{\prime}}\left(\mathbb{R}_{-}^{3}\right)$ with respect to the norm

$$
|f|_{-1, q^{\prime}, \mathbb{R}_{-}^{3}} \equiv \sup _{\|\nabla \varphi\|_{q, \mathbb{R}_{-}^{3}=1}}\left|\int_{\mathbb{R}^{3}} f \varphi\right| .
$$

Actually, the completition of this class of functionals in the negative norm is isomorphic and isometric to $D_{0}^{-1, q^{\prime}}\left(\mathbb{R}_{-}^{3}\right)$ (see [6]).

Once these definitions are given, since $\boldsymbol{w}$ belongs to $C_{0}^{\infty}\left(\overline{\mathbb{R}^{3}}\right)$, by recalling (4) and Remark 2 and 3 , it is easy to see that for all $q \in(6 / 5,6)$ and $r \in[1,2]$

$$
G \in L^{q}\left(\mathbb{R}_{-}^{3}\right), \quad F \in D_{0}^{-1, q}\left(\mathbb{R}_{-}^{3}\right) \cap L^{r}\left(\mathbb{R}_{-}^{3}\right) .
$$

Indeed, $F$ and $G$ are smooth in any bounded domain $K$, such that $\bar{K} \subset \mathbb{R}_{-}^{3}$.

REMARK 9. - When the flux $\Phi$ is zero, we have $\boldsymbol{b}=\boldsymbol{w}$ in (25). Thus, $G$ and $F$ have compact support and we can immediately infer from [4] the expected decay $O\left(|\boldsymbol{x}|^{-2}\right)$.

Now, we recall the main contents of [4] and generalize it to our case. We establish results on problem (26) admitting that the force has no compact support.

Lemma 5. - Given $F \in D_{0}^{-1,3 / 2}\left(\mathbb{R}_{-}^{3}\right)$ there exists $c>0$, such that, if

$$
|F|_{-1,3 / 2} \leqslant c v^{2} \text {, }
$$

then problem (26) admits a unique solution $\left(\tilde{\boldsymbol{u}}_{0}, \tilde{\pi}\right)$ verifying

$$
\left(\widetilde{\boldsymbol{u}}_{0}, \tilde{\boldsymbol{\pi}}\right) \in\left(D_{0}^{1,3 / 2}\left(\mathbb{R}_{-}^{3}\right) \cap L^{3}\left(\mathbb{R}_{-}^{3}\right)\right) \times L^{3 / 2}\left(\mathbb{R}_{-}^{3}\right) .
$$

The next result states that, with further hypotheses on $F, \widetilde{\boldsymbol{u}}_{0}$ also is a $D$-solution, i.e. a solution such that $\nabla \widetilde{\boldsymbol{u}}_{0} \in L^{2}\left(\mathbb{R}_{-}^{3}\right)$.

LEMMA 6. - Let $F$ and $\widetilde{\boldsymbol{u}}_{0}$ be as in Lemma 5. If moreover $F \in C^{\infty}(K)$ for any bounded domain $K$, such that $\bar{K} \subset \mathbb{R}_{-}^{3}$ and a function $G \in L^{3}\left(\mathbb{R}_{-}^{3}\right)$ exists such that $F=\nabla \cdot G$, then $\nabla \tilde{\boldsymbol{u}}_{0} \in L^{2}\left(\mathbb{R}_{-}^{3}\right), \tilde{\boldsymbol{u}}_{0} \in L^{6}\left(\mathbb{R}_{-}^{3}\right)$ and $\tilde{\boldsymbol{u}}_{0}$ fulfils the energy equality

$$
v \int_{\mathbb{R}_{-}^{3}}\left|\nabla \widetilde{\boldsymbol{u}}_{0}\right|^{2}=-\left(\nabla \widetilde{\boldsymbol{u}}_{0}, G\right) .
$$

Proof. - Lemma 6 is a generalization of Lemma 8 of [4]. In [4], $F$ has compact support; however our hypotheses on $F$ ensure that, integrating by parts the equation of motion multiplied by $\tilde{\boldsymbol{u}}_{0}$, we obtain the right-hand side of (29).

REMARK 10. - The solution $\widetilde{\boldsymbol{u}}_{0}$ decays better than a general $D$-solution, for which (29) does not hold. 
Lemma 9 of [4], where $F$ has compact support, can be extended as follows.

LEMMA 7. - Let $F$ be as in the previous Lemma. If moreover $F \in L^{3 / 2}\left(\mathbb{R}_{-}^{3}\right)$, then $\nabla \tilde{\boldsymbol{u}}_{0} \in L^{3}\left(\mathbb{R}_{-}^{3}\right)$ and $\tilde{p} \in L^{3}\left(\mathbb{R}_{-}^{3}\right)$.

Proof. - Since $F$ in $L^{3 / 2}\left(\mathbb{R}_{-}^{3}\right)$ is sufficient to apply Cattabriga's local estimates, the proof can exactiy be performed as in Lemma 9 of [4].

On the other hand, other $D$-solutions of problem (26) possibly can exist. For instance, the solution $\left(\boldsymbol{u}_{0}, p\right)$ constructed at the beginning of this section decomposing $\boldsymbol{u}$ as $\boldsymbol{u}_{0}+\boldsymbol{w}$ is such that $\nabla \boldsymbol{u}_{0} \in L^{2}\left(\mathbb{R}_{-}^{3}\right)$ and $p \in L^{2}\left(\mathbb{R}_{-}^{3}\right)$. For such a solution there exists an energy inequality analogous to (29). Precisely:

LEMMA 8. - Let $\left(\boldsymbol{u}_{0}, p\right)$ be the solution of problem (26) constructed at the beginning of this section. Then

$$
v \int_{\mathbb{R}_{-}^{3}}\left|\nabla \boldsymbol{u}_{0}\right|^{2} \leqslant-\left(\nabla \boldsymbol{u}_{0}, G\right) .
$$

PROOF. - We rewrite the energy inequality (10) with $l=0$, recalling that $\boldsymbol{u}=\boldsymbol{u}_{0}+$ $+w$, and integrate by parts. So we obtain

$$
\begin{aligned}
v \int_{\mathbb{R}_{-}^{3}}\left|\nabla \boldsymbol{u}_{0}\right|^{2} \leqslant & -v\left(\nabla \boldsymbol{u}_{0}, \nabla \boldsymbol{w}+\nabla \boldsymbol{a}\right)+\left(\boldsymbol{u}_{0} \cdot \nabla \boldsymbol{u}_{0}, \boldsymbol{a}\right)+\left(\boldsymbol{w} \cdot \nabla \boldsymbol{u}_{0}, \boldsymbol{a}\right)+ \\
& +\left(\boldsymbol{a} \cdot \nabla \boldsymbol{u}_{0}, \boldsymbol{a}\right)+v\left(\Delta\left(\boldsymbol{w}+\boldsymbol{u}_{0}+\boldsymbol{a}\right), \boldsymbol{w}\right)-(\boldsymbol{w} \cdot \nabla \boldsymbol{a}, \boldsymbol{w})-(\boldsymbol{a} \cdot \nabla \boldsymbol{a}, \boldsymbol{w})+ \\
& -\left(\boldsymbol{u}_{0} \cdot \nabla \boldsymbol{a}, \boldsymbol{w}\right)+\int_{\Sigma(0)}\left(-p w_{3}-\frac{1}{2} \boldsymbol{w}^{2}\left(a_{3}+w_{3}\right)\right) .
\end{aligned}
$$

In what follows, we use the fact that $(\boldsymbol{g} \cdot \nabla \boldsymbol{w}, \boldsymbol{w})=0$ for any solenoidal vector field $\boldsymbol{g}$ and that $\left(\boldsymbol{w} \cdot \nabla \boldsymbol{u}_{0}, \boldsymbol{u}_{0}\right)=0$, because $\boldsymbol{w}$ is zero outside the bounded domain $\Omega^{\prime}$.

On the other hand, if we multiply $\left(1_{1}\right)$ by $\boldsymbol{w}$, integrate over $\Omega^{\prime}$, and substitute the result in (31) this last reduces to

$$
\begin{aligned}
v \int_{\mathbb{R}_{-}^{3}}\left|\nabla \boldsymbol{u}_{0}\right|^{2} \leqslant-v\left(\nabla \boldsymbol{u}_{0}, \nabla \boldsymbol{w}+\nabla \boldsymbol{a}\right)-\left(\boldsymbol{u}_{0} \cdot \nabla \boldsymbol{u}_{0}, \boldsymbol{a}\right)+\left(\boldsymbol{w} \cdot \nabla \boldsymbol{u}_{0}, \boldsymbol{a}\right)+\left(\boldsymbol{a} \cdot \nabla \boldsymbol{u}_{0}, \boldsymbol{a}\right)+ \\
+\left(\boldsymbol{w} \cdot \nabla \boldsymbol{u}_{0}, \boldsymbol{w}\right)+\left(\boldsymbol{u}_{0} \cdot \nabla \boldsymbol{u}_{0}, \boldsymbol{w}\right)+\left(\boldsymbol{a} \cdot \nabla \boldsymbol{u}_{0}, \boldsymbol{w}\right)-\frac{1}{2} \int_{\Sigma(0)} \boldsymbol{w}^{2}\left(a_{3}+w_{3}\right) .
\end{aligned}
$$

A new integration by part leads to

$$
\begin{aligned}
v \int_{\mathbb{R}_{-}^{3}}\left|\nabla \boldsymbol{u}_{0}\right|^{2} \leqslant-v\left(\nabla \boldsymbol{u}_{0}, \nabla \boldsymbol{w}+\nabla \boldsymbol{a}\right)- & \left(\boldsymbol{u}_{0} \cdot \nabla \boldsymbol{u}_{0}, \boldsymbol{a}\right)+\left(\boldsymbol{w} \cdot \nabla \boldsymbol{u}_{0}, \boldsymbol{a}\right)+\left(\boldsymbol{a} \cdot \nabla \boldsymbol{u}_{0}, \boldsymbol{a}\right)+ \\
& +\left(\boldsymbol{u}_{0} \cdot \nabla \boldsymbol{u}_{0}, \boldsymbol{w}\right)+\left(\boldsymbol{w} \cdot \nabla \boldsymbol{u}_{0}, \boldsymbol{w}\right)+\left(\boldsymbol{a} \cdot \nabla \boldsymbol{u}_{0}, \boldsymbol{w}\right) .
\end{aligned}
$$


Now, if we explicite $-\left(\nabla \boldsymbol{u}_{0}, G\right)$, we obtain the terms on the right-hand side of (32). In fact the term $\left(\boldsymbol{a} \cdot \nabla \boldsymbol{u}_{0}, \boldsymbol{u}_{0}\right)$ vanishes, because $\boldsymbol{u}_{0}^{2} \boldsymbol{a} \in L^{q}\left(\mathbb{R}_{-}^{3}\right)$ with $q<3$. As is known, we can find a diverging sequence $\left\{R_{n}\right\}_{n \in \mathbb{N}}$ of radii on which $\lim _{n \rightarrow \infty} \int_{S\left(R_{n}\right)} \boldsymbol{u}_{0} \boldsymbol{a} \cdot \boldsymbol{n} d S$, where $S\left(R_{n}\right)$ is the surface of the halfsphere and $n$ the outer normal of it.

Lemma 8 permits us to show that this solution is unique (i.e. that $\left(\boldsymbol{u}_{0}, p\right)$ and $\left(\tilde{\boldsymbol{u}}_{0}, \tilde{p}\right)$ coincide) following without any changes the proof of Theorem 13 in [4].

Lemma 9. - Let $F$ be as in Lemma 7 and $G \in L^{2}\left(\mathbb{R}_{-}^{3}\right)$. If moreover

$$
|F|_{-1,3 / 2}<c^{\prime} v^{2},
$$

then the solution $\left(\tilde{\boldsymbol{u}}_{0}, \tilde{p}\right)$ of problem (26) found in Lemma 5 is unique in the class of solutions having finite Dirichlet integral and satisfying (30).

At this point we can give a first pointwise estimate of the solution, which is a generalization of Theorem 9 of [4].

It will be clear from the proof, that the most general result on the decay of solutions to problem (26), should be obtained by taking $F$ in weighted spaces (see [2]). On the other hand, our force $F$ is dependent on the solution $\boldsymbol{u}_{0}$. Thus, instead of giving general statements we deal with $F$ as in definition (25).

LEMMA 10. - Let F be defined by (25) and satisfy (27) and (28), then the D-solution of problem (26) is such that

$$
\left|\boldsymbol{u}_{0}(\boldsymbol{x})\right|=O\left(|\boldsymbol{x}|^{-1 / 2}\right) \quad \text { as }|\boldsymbol{x}| \rightarrow+\infty .
$$

Proof. - Let us define $B_{-}^{R} \equiv \mathbb{R}_{-}^{3} \backslash B_{R}(0)$, where $B_{R}(0)$ is the ball centered in 0 whose radius is $R$.

In [4, Theorem 9] Cattabriga's estimates in a ball together with classical embedding theorems, are used to prove that for $R$ large enough and $|\boldsymbol{x}| \in(R, R+1)$

$$
\left|\boldsymbol{u}_{0}(\boldsymbol{x})\right| \leqslant c\left\|\nabla \boldsymbol{u}_{0}\right\|_{2, B^{R_{-}}} .
$$

In the same proof from Saint-Venant estimates it follows that

$$
\left\|\nabla \boldsymbol{u}_{0}(\boldsymbol{x})\right\|_{2, B \underline{R}} \leqslant c|\boldsymbol{x}|^{-1 / 2} .
$$

For our problem inequality (33) becomes

$$
\left|\boldsymbol{u}_{0}(\boldsymbol{x})\right| \leqslant c\left(\left\|\nabla \boldsymbol{u}_{0}\right\|_{2, B \underline{R}}+\|F\|_{2, B \underline{R}}\right) .
$$

From (25), by Hölder and Sobolev inequalities we get

$$
\|F\|_{2, B_{-}^{R}} \leqslant c\left(\|\Delta \boldsymbol{b}\|_{2, B_{-}^{R}}+\|\boldsymbol{b} \cdot \nabla \boldsymbol{b}\|_{2, B_{-}^{R}}+\left\|\nabla \boldsymbol{u}_{0}\right\|_{2, B_{\underline{-}}^{R}}\right),
$$

where the first two terms on the right-hand side are clearly less than $c R^{-1 / 2}$, because of the pointwise decay of $|\boldsymbol{b}(\boldsymbol{x})|$ and of its derivatives. 
Thus, we are left with the problem of showing the inequality $(34)\left({ }^{3}\right)$.

To this end we multiply the equation of motion (26) by $\boldsymbol{u}_{0}$ and integrate by parts over $B_{-}^{R}$, getting

$$
v\left\|\nabla \boldsymbol{u}_{0}\right\|_{2, B \underline{R}}^{2}=\int_{\partial B_{\underline{R}}^{R}}\left(\nu \nabla \boldsymbol{u}_{0} \cdot \boldsymbol{u}_{0}-p_{0} \boldsymbol{u}_{0}-\frac{\boldsymbol{u}_{0}^{2}}{\mathbf{2}} \boldsymbol{u}_{0}+G \cdot \boldsymbol{u}_{0}\right) \cdot \boldsymbol{n}-\int_{B^{R}} G \cdot \nabla \boldsymbol{u}_{0} .
$$

The scheme of Saint-Venant technique is the following. Set

$$
\Psi(R)=v \int_{B_{-}^{R}}\left|\nabla \boldsymbol{u}_{0}\right|^{2},
$$

so that $\Psi^{\prime}(R)<0$. Integrate both sides of (36) over $R \in(\bar{R}, \infty)$ and show that $\Psi(R) \in L^{1}(\bar{R}, \infty)$.

Hence, as is known

$$
\left.\begin{array}{l}
\Psi^{\prime}(R)<0 \\
\Psi(R) \in L^{1}(\bar{R}, \infty)
\end{array}\right\} \Rightarrow \Psi(R) \leqslant c R^{-1}
$$

and this last is what we want to prove.

The first term on the right hand side of (36) is in $L^{1}$ for the summability properties of the solution. Consequently, we need only to prove $\left({ }^{4}\right)$

$$
\int_{B R} G \cdot \nabla u_{0} \in L^{1}(\bar{R}, \infty),
$$

We get this by recalling definition (25) for $G$ and using the inequalities

$$
\left\{\begin{array}{l}
\int_{B_{-}^{R}}\left|\nabla \boldsymbol{b} \cdot \nabla \boldsymbol{u}_{0}\right| \leqslant c\left\|\nabla \boldsymbol{u}_{0}\right\|_{2, \mathbb{R}_{-}^{3}} R^{-3 / 2}, \\
\int_{B_{-}^{R}}\left|\boldsymbol{b} \cdot \nabla \boldsymbol{u}_{0} \cdot \boldsymbol{b}\right| \leqslant c\left\|\nabla \boldsymbol{u}_{0}\right\|_{2, \mathbb{R}_{-}^{3}} R^{-5 / 2}, \\
\int_{B_{-}^{R}}\left|\boldsymbol{u}_{0} \cdot \nabla \boldsymbol{u}_{0} \cdot \boldsymbol{b}\right| \leqslant c\left\|\boldsymbol{u}_{0}\right\|_{3, \mathbb{R}_{-}^{3}}\left\|\nabla \boldsymbol{u}_{0}\right\|_{3 / 2, \mathbb{R}_{-}^{3}} R^{-2},
\end{array}\right.
$$

which can be deduced by the pointwise decay of $\boldsymbol{b}(\boldsymbol{x})$.

Now we recall the following result of [4, Theorem 7].

${ }^{(3)}$ Notice that, in the general case, it could be sufficient to take $F$ such that, for some $\beta \geqslant 1 / 2$ and some $\bar{R}>0,|\boldsymbol{x}|^{\beta} F(\boldsymbol{x}) \in L^{2}\left(B_{-}^{\bar{R}}\right)$.

$\left({ }^{4}\right)$ As before, it would be sufficient that for some $\beta>1$ and some $q \in[3 / 2,3]$ : $|\boldsymbol{x}|^{\beta} G(\boldsymbol{x}) \in L^{Q}\left(B_{\bar{R}}\right)$. 
Lemma 11. - Let $F \in D_{0}^{-1, q}\left(\mathbb{R}_{-}^{3}\right) \cap D_{0}^{-1,3 / 2}\left(\mathbb{R}_{-}^{3}\right), q \in(1,3)$. There exist $c, c^{\prime \prime}>0$ such that, if

$$
|F|_{-1,3 / 2}<\min \left\{c v^{2}, c^{\prime \prime} \nu\right\},
$$

then problem (26) admits a unique solution $\left(\widetilde{\boldsymbol{u}}_{0}, \widetilde{p}\right)$ verifying

$$
\nabla \widetilde{\boldsymbol{u}}_{0} \in L^{q}\left(\mathbb{R}_{-}^{3}\right) \cap L^{3 / 2}\left(\mathbb{R}_{-}^{3}\right) \quad \text { and } \quad \tilde{p} \in L^{q}\left(\mathbb{R}_{-}^{3}\right) \cap L^{3 / 2}\left(\mathbb{R}_{-}^{3}\right) .
$$

REMARK 11. - If $F$ verifies certain smallness assumptions (see (38)), all the previous lemmas, together with the convexity inequality and the Sobolev embedding theorem [6], state that the $D$-solution satisfying the energy equality is unique and

$$
\begin{array}{rlrl}
p, \nabla \boldsymbol{u}_{0} & \in L^{q}\left(\mathbb{R}_{-}^{3}\right), & \forall q \in(6 / 5,3], \\
\boldsymbol{u}_{0} \in L^{q}\left(\mathbb{R}_{-}^{3}\right), & \forall q \in(2,+\infty) .
\end{array}
$$

Hence $F \in D_{0}^{-1, q}\left(\mathbb{R}_{-}^{3}\right), G \in L^{q}\left(\mathbb{R}_{-}^{3}\right) \forall q \in(1,+\infty)$. So Lemma 11 gives $\boldsymbol{u}_{0} \in L^{q}\left(\mathbb{R}_{-}^{3}\right)$ $\forall q \in(3 / 2,+\infty)$.

We set $K=\min \left\{c v^{2}, c^{\prime} v^{2}, c^{\prime \prime} v\right\}$.

All the results on the regularity lead to the following.

THEOREM 2. - Let $F$ be defined as in (26). There exists a constant $K$, depending on $v$, such that, if

$$
|F|_{-1,3 / 2} \leqslant K
$$

then, as $|\boldsymbol{x}| \rightarrow+\infty$, the $D$-solution $\boldsymbol{u}_{0}$ of problem (26) verifies:

$$
\begin{aligned}
& \text { if } \Phi \neq 0, \quad\left|\boldsymbol{u}_{0}(\boldsymbol{x})\right|=O\left(\frac{\ln |\boldsymbol{x}|}{|\boldsymbol{x}|^{2}}\right), \\
& \text { if } \Phi=0, \quad\left|\boldsymbol{u}_{0}(\boldsymbol{x})\right|=O\left(|\boldsymbol{x}|^{-2}\right) .
\end{aligned}
$$

Proof. - By virtue of Remark 9, the proof concerns only the case $\Phi \neq 0$. First of all we note that

$$
|G(\boldsymbol{x})|=O\left(|\boldsymbol{x}|^{-5 / 2}\right) \quad \text { as }|\boldsymbol{x}| \rightarrow+\infty .
$$

This property of $G$ can be inferred from (25) using Lemma 10. In this way, considering the representation

$$
\boldsymbol{u}_{0}(\boldsymbol{x})=-\int_{\mathbb{R}_{-}^{3}} \nabla_{y} \mathfrak{S}(\boldsymbol{x}, \boldsymbol{y}) \cdot\left(\left(\boldsymbol{u}_{0} \otimes \boldsymbol{u}_{0}\right)(\boldsymbol{y})-G(\boldsymbol{y})\right) d \boldsymbol{y},
$$

where $\mathcal{G}(\boldsymbol{x}, \boldsymbol{y})$ is the Green's tensor for the Stokes problem, we can repeat the proof of 
Theorem 10 given in [4] for the term

$$
\int_{\mathbb{R}_{-}^{3}} \nabla_{y} \mathcal{G}(\boldsymbol{x}, \boldsymbol{y}) \cdot\left(\boldsymbol{u}_{0} \otimes \boldsymbol{u}_{0}\right)(\boldsymbol{y}) d \boldsymbol{y},
$$

recalling that $\left|\nabla_{y} \mathfrak{G}(\boldsymbol{x}, \boldsymbol{y})\right| \leqslant \boldsymbol{c} /\left(|\boldsymbol{x}-\boldsymbol{y}|^{2}\right)$ and Remark 11. As far as

$$
\int_{\mathbb{R}_{-}^{3}} \nabla_{y} \mathcal{S}(\boldsymbol{x}, \boldsymbol{y}) \cdot G(\boldsymbol{y}) d \boldsymbol{y}
$$

is concerned we recall (25), the asymptotic properties of $\boldsymbol{b},|\nabla \boldsymbol{b}|$ and proceed as in Theorem 10 of [4], choosing $|x|=2 R$.

The only difference relates to

$$
I \equiv \int_{B_{R_{-}}} \frac{|\nabla \boldsymbol{b}(\boldsymbol{y})|}{|\boldsymbol{x}-\boldsymbol{y}|^{2}} d \boldsymbol{y},
$$

where $B_{R_{-}} \equiv B_{R}(0) \cap \mathbb{R}_{-}^{3}$. If we choose $\bar{R}$ such that $1<\bar{R}<R$, then

$$
B_{R_{-}}=\left(B_{\bar{R}}(0) \cap \mathbb{R}_{-}^{3}\right) \cup\left(B_{R_{-}} \backslash\left(B_{\bar{R}}(0) \cap \mathbb{R}_{-}^{3}\right)\right),
$$

so that

$$
I \leqslant \frac{c}{R^{2}}+\int_{B_{R_{-}} \backslash B_{\bar{R}}(0)} \frac{d \boldsymbol{y}}{|\boldsymbol{y}|^{3}|\boldsymbol{x}-\boldsymbol{y}|^{2}} .
$$

But $|\boldsymbol{x}-\boldsymbol{y}| \geqslant R$ in $B_{R_{-}}$and hence

$$
l_{1} \equiv \int_{B_{R_{-}} \backslash B_{\bar{R}}(0)} \frac{d \boldsymbol{y}}{|\boldsymbol{y}|^{3}|\boldsymbol{x}-\boldsymbol{y}|^{2}} d \boldsymbol{y} \leqslant \frac{R^{\varepsilon}}{\varepsilon R^{2}}, \quad \forall \varepsilon>0 .
$$

Taking into account that $R^{\varepsilon} / \varepsilon$ has the absolute minimum $e \ln R$ for $\varepsilon \in(0,+\infty)$ we deduce

$$
I_{1} \leqslant \frac{e \ln R}{R^{2}}
$$

From that the thesis follows.

REMARK 12. - All smallness assumptions of $|F|_{-1,3 / 2}$ can be reformulated in terms of the flux $\Phi$. Since

$$
|F|_{-1,3 / 2} \leqslant\|G\|_{3 / 2},
$$

by the definition of $G$ and using the properties of the flux carrier $a$, it can be shown that $|F|_{-1,3 / 2}$ can be increased by powers of $|\Phi|$. The estimate is obtained, recalling that

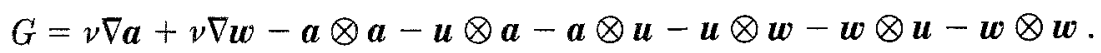


In order to estimate $\boldsymbol{u}$, it is sufficient to recall (9) with $l=0$ while, to estimate $\boldsymbol{w}$ we have to put together (24) and (9). In particular, using Gagliardo's theorem and the Poincaré inequality we have

$$
\left\|\boldsymbol{u}^{*}\right\|_{1 / 2,2, \partial \Omega^{\prime}} \leqslant c\|\nabla \boldsymbol{u}\|_{2, \Omega^{\prime}},
$$

so that condition (28) becomes

$$
\|\boldsymbol{w}\|_{2, \Omega^{\prime}} \leqslant c\|\nabla \boldsymbol{w}\|_{2, \Omega^{\prime}} \leqslant c\|\nabla u\|_{2, \mathrm{R}_{-}^{3}} \leqslant P_{0}(|\Phi|),
$$

where $P_{0}(|\Phi|)$ is a polynomial in $|\Phi|$ of 10th degree, without the constant term.

The estimate of $G$ is obtained by means of the Hölder inequality. Consider, for instance, the term $\boldsymbol{u} \otimes \boldsymbol{w}$ :

$$
\left(\int_{R_{-}^{3}}|\boldsymbol{u} \otimes \boldsymbol{w}|^{3 / 2}\right)^{2 / 3} \leqslant\|\boldsymbol{u}\|_{6}\|\boldsymbol{w}\|_{2} .
$$

Thus, we finally get

$$
\|G\|_{3 / 2} \leqslant P(|\Phi|),
$$

where $P(|\Phi|)$ is again polynomial, but of 20 th degree. So, if we take small $|\Phi|$ the sufficient condition of Theorem 2 can be replaced by

$$
P(\Phi)<c_{0}|\Phi|<K,
$$

where $K$ is given in Theorem 2 .

Case $\alpha \neq 0$ : Let us consider directly the restriction of problem (PM) to $\mathbb{R}_{-}^{3}$; we find a problem with compact support data on the boundary and zero body force. For this we have that the solution $(\boldsymbol{v}, p)$ has the summability properties found in [10]:

$$
\begin{aligned}
& v \in W^{2, q}\left(\mathbb{R}_{-}^{3}\right), \quad \nabla p \in L^{q}\left(\mathbb{R}_{-}^{3}\right), \quad \forall q \in(3 / 2,+\infty), \quad \text { if } \Phi \neq 0, \\
& v \in W^{2, q}\left(\mathbb{R}_{-}^{3}\right), \quad \nabla p \in L^{q}\left(\mathbb{R}_{-}^{3}\right), \quad \forall q \in(1,+\infty), \quad \text { if } \Phi=0 .
\end{aligned}
$$

Thus, we have only to summarize the results of [10], which hold true without any restriction on the size of $|\Phi|$.

THEOREM 3. - Let $\boldsymbol{v}(\boldsymbol{x})$ be the D-solution of problem (PM). Then everywhere $\mathbb{R}_{-}^{3}$, with exception of the exterior of a cone of arbitrary aperture, whose axis is $x_{3}$ and whose vertex in the origin,

$$
\begin{aligned}
& |\boldsymbol{v}(\boldsymbol{x})|=O\left(|\boldsymbol{x}|^{-1+\varepsilon}\right), \quad \forall \varepsilon>0, \quad \text { if } \Phi \neq 0, \\
& |\boldsymbol{v}(\boldsymbol{x})|=O\left(|\boldsymbol{x}|^{-3+\varepsilon}\right), \quad \forall \varepsilon>0, \quad \text { if } \Phi=0,
\end{aligned}
$$

as $|\boldsymbol{x}| \rightarrow+\infty$. 
Acknowledgments. The authors thank Professor G. P. GaLdI and Professor M. R. PADULA for helpful discussions.

This work was supported by the Italian M.U.R.S.T. (60\% contract at the University of Ferrara) and the German DFG (50\% contract at the University of Paderborn). It was started during the stay of G. THÄTER in Ferrara, that was subsidized by the Cassa di Risparmio di Ferrara.

\section{REFERENCES}

[1] M. E. BoGovskiI, Solution of the first boundary value problem for the equation on continuity of an incompressible medium, Sov. Math. Dokl., 20 (1979), pp. 1094-1098.

[2] W. Borchers - K. PILECKas, Existence, Uniqueness and Asymptotics of Steady Jets, Arch. Rational Mech. Anal., 120 (1992), pp. 1-49.

[3] H. ChANG, Speedy jets into a halfspace govermed by the Navier-Stokes equations, Proc. IUTAM Symp. 1991, Springer-Verlag, Lecture Notes in Mathematics, 1530 (1992), pp. 85-96.

[4] V. Coscia - M. C. PATRIA, Existence, uniqueness and asymptotic decay of steady imcompressible flows in a halfspace, Stud. Appl. Anal. Cont. Media, 2 (1992), pp. 111-131.

[5] R. FARWIG - H. SOHR, An Approach to Resolvent Estimates for the Stokes Equations in $L^{q_{-}}$ spaces, Proc. IUTAM Symp. 1991, Springer-Verlag, Lecture Notes in Mathematics, 1530 (1992), pp. 97-110.

[6] G. P. GALDI, An introduction to the mathematical theory of the Navier-Stokes equations: Volume 2, Non-linear steady problems, Springer Tracts in Natural Philosophy (1994).

[7] J. G. HEYwoon, The Navier-Stokes equations: on the existence, regularity and decay of solutions, Indiana Univ. Math. J., 29 (1980), pp. 639-681.

[8] O. A. LadyzhenskaYa - V. A. Solonnikov, Determination of the solutions of boundry value problems for stationary Stokes and Navier-Stokes equations having an unbounded Dirichlet integral, translated from Zapiski nauchnykh Seminarov Leningradskogo Otdeleniya Matematicheskogo Instituta im. V. A. Steklova Akas. Nauk USSR, 96 (1980), pp. 117-160.

[9] P. MaRemonti, Some theorems of existence for solutions of the Navier-Stokes equations with slip conditions in half-space, Ricerche Mat., 40 (1991), pp. 81-135.

[10] A. PASSERINI, Steady flows of a viscous incompressible fluid in a porous half-space, $\mathrm{M}^{3} \mathrm{AS}$, 4 (1994), pp. 705-732. 